\title{
Paricalcitol supplementation during the first year after kidney transplantation does not affect calcification propensity score
}

\author{
Amin Ussif ${ }^{1 *}$, Hege Pihlstrøm ${ }^{1}$, Andreas Pasch², Hallvard Holdaas ${ }^{1}$, Anders Hartmann $^{1,3}$, Knut Smerud $^{4}$ \\ and Anders Åsberg ${ }^{1,5,6}$
}

\begin{abstract}
Background: Cardiovascular complications are common in kidney transplant patients and calcification propensity of blood, measured as $T_{50}$, is associated with cardiovascular outcomes. Paricalcitol supplementation affects calcium/ phosphate homeostasis and may affect calcification propensity. To assess this hypothesis we measured $T_{50}$ in kidney transplant recipients participating in a randomized study comparing paricalcitol versus no treatment during the first year after kidney transplantation.

Methods: Stored serum samples from 76 kidney transplant recipients (paricalcitol $n=37$, no treatment $n=39$ ) were analyzed. Analyses were performed at inclusion ( 8 weeks after transplantation) and repeated one year after transplantation.

Results: There were no statistically significant differences in $T_{50}$ between the paricalcitol and placebo groups, neither at baseline $(p=0.56)$ nor at 1 year $(p=0.61)$. Also, there were no significant changes in $T_{50}$ over time in either group or when pooling all data $(p<0.20)$. In multivariate regression analysis, out of 16 potentially relevant covariates, comprising clinical and biochemical parameters, only plasma PTH and $T_{50}$ at baseline were significantly correlated to $T_{50}$ after one year. ( $p<0.03$ and $p<0.01$, respectively).

Conclusions: Calcium propensity measured as $T_{50}$ score remained unchanged with paricalcitol treatment in kidney transplant recipients, and was not changed over time during the study period of one year.
\end{abstract}

Trial registration: ClinicalTrials.gov, NCT01694160, registered 23 September 2012.

Keywords: Paricalcitol, Calcification propensity score, Randomized controlled trial, Renal transplantation

\section{Background}

Patients with chronic kidney disease have increased risk of cardiovascular complications and impaired survival [1]. Vascular calcification is a prominent feature in chronic kidney disease (CKD) patients that is also associated with cardiovascular morbidity and mortality $[2,3]$. Restoration of kidney function following kidney transplantation seems to alleviate the burden of cardiovascular complications, but the risk remains substantially increased compared with the general population $[4,5]$.

\footnotetext{
* Correspondence: aussif@hotmail.com

1 Department of Transplantation Medicine, Oslo University Hospital

Rikshospitalet, P.O. Box 4950, Nydalen, 0424 Oslo, Norway

Full list of author information is available at the end of the article
}

Calcium/phosphate homeostasis undergoes substantial changes following transplantation. There have been some indications that low 25-hydroxyvitamin D (25-OH)D may be associated with proteinuria and fibrosis in renal transplant recipients [6, 7]. Paricalcitol (19-nor-1,25-dihydroxyvitamin D2) is a synthetic, selective third generation vitamin $\mathrm{D}$ receptor agonist associated with low risk of hypercalcaemia $[8,9]$. In CKD, paricalcitol effectively and safely suppresses PTH and may reduce proteinuria. Similar effects, although less established, have been suggested in renal transplant recipients [10].

We therefore recently performed a randomized controlled trial (RCT) comparing paricalcitol and no treatment to assess effects on albuminuria, inflammation related gene expression profiles and fibrosis in protocol

(c) The Author(s). 2018 Open Access This article is distributed under the terms of the Creative Commons Attribution 4.0 International License (http://creativecommons.org/licenses/by/4.0/), which permits unrestricted use, distribution, and 
biopsies [10]. The study did not reveal any effects on these parameters, but plasma parathyroid hormone (PTH) levels were as expected reduced in the active treatment group. We speculated that the effects on PTH and calcium/phosphate might influence the calcification propensity of the patients, which can be measured in serum by a novel test [11]. The test readout $\left(\mathrm{T}_{50}\right)$ reflects the functionality of a physiologic system, the system of humoral calcification control, by measuring the time of transformation of soluble primary calciprotein particles (CPPs) to secondary, crystalline (hydroxyapatite-containing) CPPs during stimulation of calcification in vitro. $\mathrm{T}_{50}$ is the time to half maximal transition to insoluble crystalline CPPs and a lower $\mathrm{T}_{50}$ signifies increased tendency for calcification. It has recently been found that $\mathrm{T}_{50}$ is a strong and independent risk factor for adverse outcomes including cardiovascular death both in CKD [12, 13] and kidney transplant recipients [14, 15].

In the present study the primary aim was to reveal any possible effect of paricalcitol supplementation on $\mathrm{T}_{50}$ scores during the first year after transplantation.

\section{Methods}

Study participants were recruited in the period of Jan 2013 to Feb 2014 from the National Transplant Centre at Oslo University Hospital, Rikshospitalet, Oslo, Norway. The original study was an open-label, randomized controlled trial comparing paricalcitol treatment to standard of care treatment in de-novo renal transplant recipients. Recipients are routinely followed for 8-10 weeks after engraftment and return for a routine one-year surveillance follow-up. The random allocation sequence was generated by an independent statistician at the monitoring facility, using computer-generated block-randomization with nonfixed block size. The principal investigator (PI) performed the opening of sealed envelopes and informed the participants of their group assignment. Treatment allocation remained undisclosed to the staff performing laboratory measurements [10]. In short, patients were invited to participate in the study in a stable phase 7-8 weeks after transplantation.

Patients receiving standard calcineurin inhibitor based triple immunosuppression with eGFR $>30 \mathrm{ml} / \mathrm{min} /$ $1.73 \mathrm{~m}^{2}$ with normocalcemia were considered for inclusion. Previous parathyroidectomy, treatment with vitamin D or calcimimetics were the main exclusion criteria. The patients were randomised to receive open label treatment with oral paricalcitol (Zemplar (Abbvie) $2 \mu \mathrm{g} /$ day) or no treatment. Serum samples were obtained at time of randomization and at one year after transplantation and stored at minus $70{ }^{\circ} \mathrm{C}$. The analysis of $\mathrm{T}_{50}$ scores was performed in one batch using laser detection of fully calcified particles as described in detail elsewhere [11].

In short, analyses were performed in triplicates in on 384-well plates. Patient serum $(40 \mathrm{uL})$ was exposed to high and supersaturated concentrations of calcium $(35 \mathrm{uL})$ and phosphate $(25 \mathrm{uL})$. The transformation step was then monitored at $37{ }^{\circ} \mathrm{C}$ using time-resolved nephelometry (bmg labtech, Ortenberg, Germany). Nonlinear regression curves were calculated, allowing the determination of the one-half maximal transition time (T50).

Adequate samples for analysis of $\mathrm{T}_{50}$ were obtained from all patients in the treatment $\operatorname{arm}(n=37)$ and all except one in the control group $(n=39)$. Demographic and transplant related data of the patients in the two groups are shown in Table 1.

\section{Statistical methods}

Baseline summary data of the total study population $(n=76)$ was calculated using descriptive analysis tool. To identify possible confounders and predictors for the subsequent analysis Pearson correlation analysis was also conducted (results not reported). Both univariate and multivariate linear regression models were employed in our analysis. Treatment, demographic and biochemical variables were included in the linear regression models. The inclusion criteria for the multiple regression models were based on clinical and biological plausibility. All analysis was performed using IBM SPSS Statistics version 24, release 24.0.0.1 and plots were done using MS Excel 2010.

The study was performed in compliance with GCP and the Helsinki declaration and approved by Norwegian Medicines Agency and the Regional Ethics committee of Health region South-East and registered on www.clinicaltrial.gov (NCT01694160 (2012/107D)).

\section{Results}

Demographic, clinical and biochemical characteristics of the population under study are summarized in Table 1. No clinically significant differences were observed between the groups at baseline. In particular, the means of $\mathrm{T}_{50}$ scores did not differ significantly at baseline; $311 \pm$ $35 \mathrm{~min}$ in the paricalcitol $316 \pm 48 \mathrm{~min}$ in the no treatment group, respectively $(p=0.56)$. Both groups were predominantly male $(78.9 \%)$ with mean age of $55 \pm 12.7$ years old and plasma PTH of $11.5 \pm 5.5 \mathrm{pmol} / \mathrm{L}$. In total $31 \%$ of patients were predialytic at time of transplantation, and $30 \%$ received organs from living donors. Only $13 \%$ were current smokers, while $45 \%$ were previous smokers. Details of the baseline characteristics are discussed in Pihlstrøm et al. [10].

Figure 1 is a graphical display of the means \pm 2 SDs of $\mathrm{T}_{50}$ scores for the paricalcitol vs no treatment group at 
Table 1 Demographics and transplant characteristics, mean (SD), at baseline (8 weeks - 1 year after renal transplantation) -

\begin{tabular}{|c|c|c|c|c|}
\hline Variables & $\begin{array}{l}\text { Paricalcitol } \\
N=37\end{array}$ & $\begin{array}{l}\text { Paricalcitol } \\
N=37\end{array}$ & $\begin{array}{l}\text { No treatment } \\
N=39\end{array}$ & $\begin{array}{l}\text { No treatment } \\
N=39\end{array}$ \\
\hline Time point post $\mathrm{Tx}$ & 8 weeks & 1 year & 8 weeks & 1 year \\
\hline Age (years) & $56.0(13.4)$ & NA & $54.2(12.1)$ & NA \\
\hline Albumin (g/L) & $42.4(2.4)$ & $43.3(2.5)$ & $41.7(2.5)$ & $43.3(2.7)$ \\
\hline $\operatorname{ALP}(\mathrm{U} / \mathrm{L})$ & $61.4(21.7)$ & $58.4(20.5)$ & $70.2(28.0)$ & $71.4(33.4)$ \\
\hline BMI $\left(\mathrm{kg} / \mathrm{m}^{2}\right)$ & $26.1(3.2)$ & NA & $25.5(3.9)$ & NA \\
\hline Calcium, total (mmol/L) & $2.4(0.1)$ & $2.4(0.1)$ & $2.4(0.1)$ & $2.4(0.1)$ \\
\hline Creatinine $(\mu \mathrm{mol} / \mathrm{L})$ & $114.4(24.5)$ & $110.3(26.7)$ & $120.9(29.6)$ & $114.6(27.5)$ \\
\hline Phosphate (mmol/L) & $0.9(0.2)$ & $0.9(0.1)$ & $0.9(0.3)$ & $0.9(0.1)$ \\
\hline PTH (pmol/L) & $11.5(5.7)$ & $7.9(4.8)$ & $11.4(5.3)$ & $9.9(3.8)$ \\
\hline $\mathrm{T}_{50}(\mathrm{~min})$ & $311(35)$ & $319(34.5)$ & 319 (34) & $323(35.6)$ \\
\hline HLA mismatch & $3.1(1.3)$ & NA & $3.0(1.4)$ & NA \\
\hline Gender (\% male) & $27(73)$ & NA & $33(84.6)$ & NA \\
\hline Smoking (\% Yes) & $5(13.5)$ & NA & $5(12.8)$ & NA \\
\hline Living donor (\% Yes) & $10(27.0)$ & NA & $13(33.3)$ & NA \\
\hline Predialytic transplantation (\% Yes) & $13(31.1)$ & NA & $12(30.8)$ & NA \\
\hline Pretransplant diabetes (\% Yes) & $7(18.9)$ & NA & $6(15.4)$ & NA \\
\hline
\end{tabular}

NA denotes not available. For clarity all figures are reported to two decimals

Abbreviations: ALP alkaline phosphatase, BMI body mass index, PTH parathyroid hormone, $\mathrm{T}_{50}$; time to $50 \%$ transfer to crystalline particles, $H L A$ human leukocyte antigen

baseline (i.e. eight weeks after transplantation) and after one year. The trend lines between the two measurement points show no significant change over time and no difference between groups based on paired t-test, ANOVA and a simple linear regression on the categorical variables (data not shown).

Table 2 shows univariate and multivariate linear regression analyses. The regression constant is not reported here for brevity. In univariate analysis, only $\mathrm{T}_{50}$ score at baseline $(p<0.01)$ is significantly associated with $\mathrm{T}_{50}$ score at one year, while baseline plasma PTH $(p=0.09)$, pretransplant dialysis $(p=0.09)$ and living donor $(p=0.08)$ are not significantly associated with $\mathrm{T}_{50}$ score at study end. The statistical significant variables in the multivariate analysis were $\mathrm{T}_{50}$ score at baseline $(p<0.01)$ and baseline PTH $(p=0.031)$. From the standardized (beta) coefficients

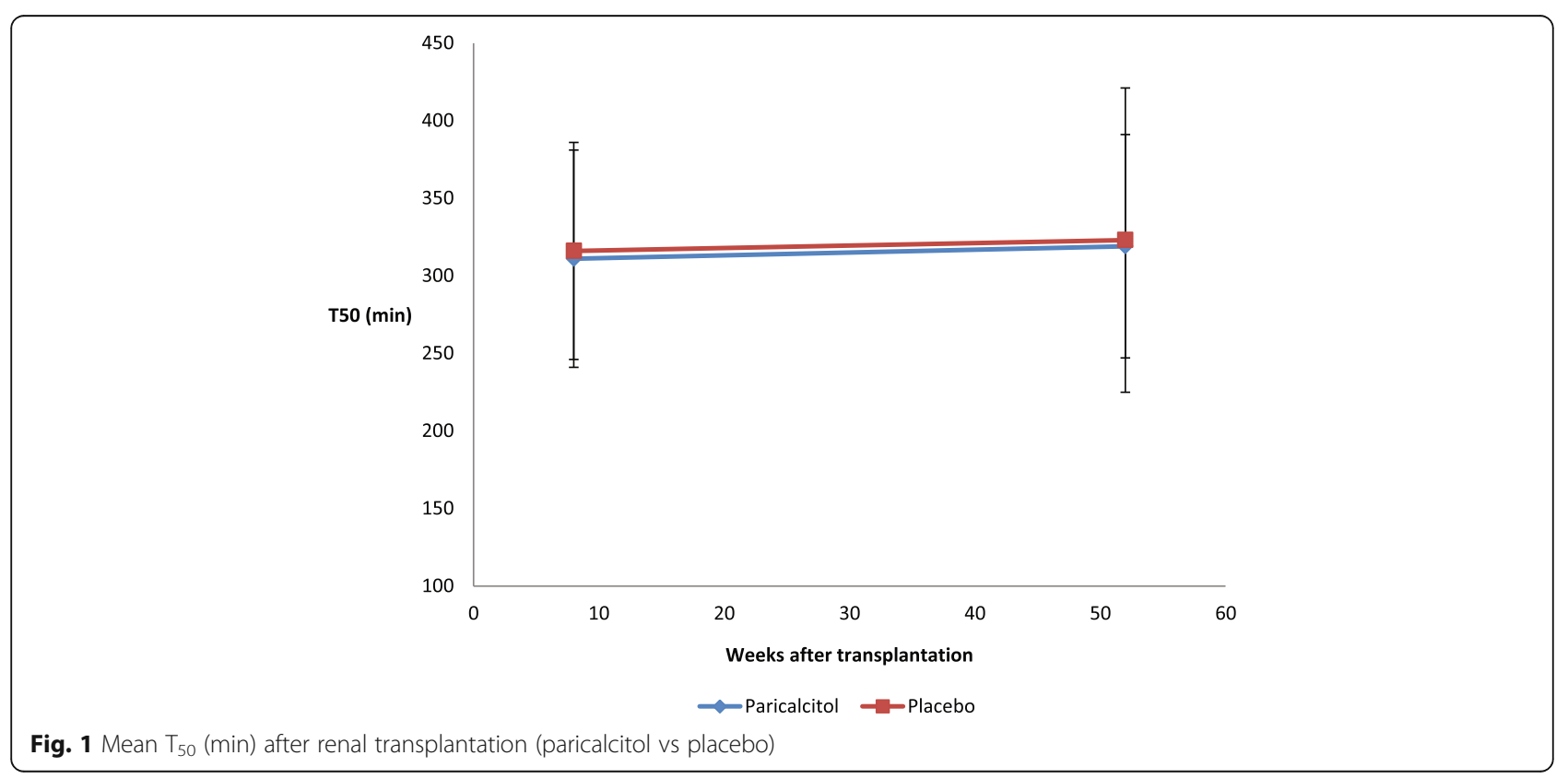


Table 2 Uni- and multivariate linear regression analysis of predictors of $T_{50}$ at one year

\begin{tabular}{|c|c|c|c|c|}
\hline \multirow[b]{2}{*}{ Variable } & \multicolumn{2}{|l|}{ Univariate } & \multicolumn{2}{|l|}{ Multivariate } \\
\hline & $\beta$ (t-values)* $^{*}$ & $p$-value & $\beta$ (t-values)* $^{*}$ & $p$-value \\
\hline Age (years) & $-0.15(-0.48)$ & 0.64 & $0.22(0.64)$ & 0.53 \\
\hline Gender (\% male) & $0.079(0.01)$ & 0.99 & $-6.30(-0.48)$ & 0.63 \\
\hline BMI $\left(\mathrm{kg} / \mathrm{m}^{2}\right)$ & $0.84(0.74)$ & 0.46 & $0.69(0.57)$ & 0.57 \\
\hline Smoking & $0.65(0.08)$ & 0.94 & $-3.12(-0.25)$ & 0.80 \\
\hline HLA mismatch & $1.06(0.36)$ & 0.72 & $0.81(0.25)$ & 0.80 \\
\hline Living donor & $15.23(1.77)$ & 0.08 & $-2.83(-0.26)$ & 0.79 \\
\hline Predialytic transplantation & $11.13(1.31)$ & 0.19 & $11.12(1.15)$ & 0.25 \\
\hline Pretransplant diabetes & $-17.65(-1.68)$ & 0.107 & $-5.02(-0.39)$ & 0.70 \\
\hline Treatment & $-4.12(-0.51)$ & 0.61 & $0.37(0.04)$ & 0.97 \\
\hline $\mathrm{T}_{50}$ baseline (min) & $0.36(4.12)$ & $<0.01$ & $0.37(3.44)$ & $<0.01$ \\
\hline Albumin (g/L) & $1.03(0.64)$ & 0.64 & $0.15(0.08)$ & 0.94 \\
\hline $\mathrm{ALP}(\mathrm{U} / \mathrm{L})$ & $0.02(0.12)$ & 0.90 & $0.18(0.95)$ & 0.35 \\
\hline Calcium (mmol/L) & $3.48(0.07)$ & 0.93 & $-6.11(-0.12)$ & 0.90 \\
\hline Creatinine $(\mu \mathrm{mol} / \mathrm{L})$ & $0.01(0.06)$ & 0.95 & $-0.01(-0.04)$ & 0.97 \\
\hline Phosphate (mmol/L) & $-4.79(-0.30)$ & 0.76 & $5.07(0.28)$ & 0.78 \\
\hline PTH (pmol/L) & $-1.61(-2.24)$ & 0.09 & $-1.70(-2.21)$ & 0.03 \\
\hline
\end{tabular}

Abbreviations: $A L P$ alkaline phosphatase, $B M I$ body mass index, $P T H$ parathyroid hormone, $T_{50}$; time to $50 \%$ transfer to crystalline particles, $H L A$ human leukocyte antigen Estimates are reported together with their t-values in parenthesis and $p$-values are in separate columns. ${ }^{*}$ t-value at $95 \%$ cutoff 1.96

the $\mathrm{T}_{50}$ score at baseline (beta $=0.43$ ) and baseline PTH (beta $=-0.25)$ are the two most important predictors. The overall multivariate regression model $(\mathrm{F}=1.48, p=0.14)$ is not statistically significant. Goodness of fit $\left(R^{2}\right)$ equals 0.29 ; hence our model explains only about $30 \%$ of that data.

\section{Discussion}

We studied the potential effects of paricalcitol supplementation during the first year after kidney transplantation on calcification propensity score in his paper. Available data show that treatment with paricalcitol did not significantly affect $T_{50}$. Calcification propensity measured as serum $\mathrm{T}_{50}$ scores at baseline and after one year of treatment with paricalcitol in a RCT was analyzed in the present study. The novel finding in the present analysis was that all analyses demonstrated that paricalcitol treatment during the first year after transplantation did not influence $\mathrm{T}_{50}$ score.

In addition to this important but negative finding, our qualitative analysis shows a flat trend in the $\mathrm{T}_{50}$ score, which is similar to earlier results reported for $T_{50}$ in kidney transplant recipients [15]. In another study, assessing the effect of ibandronate over time, $\mathrm{T}_{50}$ scores increased substantially during the first few weeks after transplantation, but then remained unchanged over the first year regardless of treatment allocation [16].

In the present original study, paricalcitol treatment was significant associated with a reduction in serum PTH [10]. However, the reduction in plasma PTH in the treatment arm did not associate with a change in $\mathrm{T}_{50}$ score. On the contrary, plasma PTH at baseline was the only clinical covariate showing independent associations with $\mathrm{T}_{50}$ score at one year. This may indicate that elevated PTH early after transplantation reflects long standing effects associated with hyperparathyroidism that may be more important than changes following normalization of kidney function during the first posttransplant year. There are several factors that influence T50 scores that are not account for in this post-hoc study. Plasma magnesium and bicarbonate are significant contributors to factors influencing $\mathrm{T}_{50}$ scores, unfortunately, these were not measured. Magnesium levels are generally low in kidney transplant recipients, especially in the early phase after transplantation. The bicarbonate concentration is first of all dependent on kidney function. Plasma magnesium may normalize within the first year whereas bicarbonate usually remains within normal values as long as the kidney function remains stable. We did not find any change in $\mathrm{T}_{50}$ over the first year that could beexplained by variations in these parameters. In any case there is no reason to believe that magnesium and bicarbonate concentrations would change differently with or without paricalcitol treatment.

\section{Strengths and limitations}

The current investigation is a post-hoc analysis that was not predetermined, which is an obvious weakness and it should be interpreted accordingly. Also the lack of effect 
of paricalcitol beyond lowering of PTH in the primary study may be considered a downside for effect studies on other parameters like $\mathrm{T}_{50}$. However, the study was indeed a properly performed RCT.

\section{Conclusions}

To summarize, treatment with paricalcitol did not have any effect on $T_{50}$ in renal transplant recipients as compared with controls without treatment. Also there were no differences in $\mathrm{T}_{50}$ scores at baseline or at one year in either group. Baseline PTH levels were significantly associated with $\mathrm{T}_{50}$ score at one year. Further studies are warranted to assess the therapeutic efficacy of interventions such as magnesium on clinical outcome measures.

\section{Abbreviations}

(25-OH)D: 25-hydroxyvitamin D; ANOVA: Analysis of variance; CKD: Chronic kidney disease; CNI: Calcineurin inhibitor; CPPs: Primary calciprotein particles; eGFR: Estimated glomerular filtration rate; PTH: Parathyroid hormone; RCT: Randomized controlled trial; SD: Standard deviation

\section{Funding}

The clinical trial was funded via internal resources at Oslo University hospital and the analysis in the current investigation was covered by budgets of the involved parties.

\section{Availability of data and materials}

The datasets generated and/or analysed during the current study are not publicly available due to Norwegian law and personal data protection regulations, but are available from the corresponding author on reasonable request.

\section{Authors' contributions}

UA participated in data analysis and writing of the article. HP participated in research design, performance of the research, data analysis and writing of the article. AP participated in measurement of calcification propensity and critical revision of the manuscript. $\mathrm{HH}$ participated in research design, performance of the research and critical revision of the manuscript. $\mathrm{AH}$ participated in research design, performance of the research, data analysis and writing of the article. KS participated in research design and critical revision of the manuscript. $A \AA$ participated in design of the research and writing of the manuscript. All authors have read and approved the final version of the manuscript.

\section{Ethics approval and consent to participate}

The study protocol was approved by the Regional Ethics Committee for the Oslo region (Health region south-east). All participants gave their written informed consent before any study-associated investigations were performed.

\section{Consent for publication}

Not applicable.

\section{Competing interests}

AP is the inventor of the $T_{50}$ test, and holds stock in Calciscon AG, Berne, Switzerland, the company that markets the $T_{50}$ test. All other authors declare no competing interest.

\section{Publisher's Note}

Springer Nature remains neutral with regard to jurisdictional claims in published maps and institutional affiliations.

\section{Author details}

'Department of Transplantation Medicine, Oslo University Hospital Rikshospitalet, P.O. Box 4950, Nydalen, 0424 Oslo, Norway. ${ }^{2}$ Calciscon AG, Bern, Switzerland. ${ }^{3}$ Institute of Clinical Medicine, Faculty of Medicine, University of Oslo, Oslo, Norway. ${ }^{4}$ Smerud Medical Research International AS, Oslo, Norway. ${ }^{5}$ The Norwegian Renal Registry, Oslo University Hospital Rikshospitalet, Oslo, Norway. 'Department of Pharmaceutical Biosciences, School of Pharmacy, University of Oslo, Oslo, Norway.

Received: 23 August 2017 Accepted: 3 August 2018

Published online: 22 August 2018

\section{References}

1. Matsushita K, van der Velde M, Astor BC, et al. Association of estimated glomerular filtration rate and albuminuria with all-cause and cardiovascular mortality in general population cohorts: a collaborative meta-analysis. Lancet. 2010;375(9731):2073-81.

2. Schlieper G, Schurgers L, Brandenburg V, Reutelingsperger C, Floege J. Vascular calcification in chronic kidney disease: an update. Nephrol Dial Transplant. 2016:31(1):31-9.

3. Jono $S$, Shioi A, Ikaria $Y$, et al. Vascular calcification in chronic kidney disease J Bone Miner Metab. 2006:24:176-81.

4. Blacher J, Guerin AP, Pannier B, Marchais SJ, London GM. Arterial calcifications, arterial stiffness, and cardiovascular risk in end-stage renal disease. Hypertension. 2001;38:938-42.

5. Hernandez D, Rufino M, Bartolomei S, et al. Clinical impact of pre-existing vascular calcfications on mortality rate after renal transplantation. Kidney Int. 2005:67:2015-20.

6. Amer $H$, Griffin MD, Stegall MD, et al. Oral paricalcitol reduces the prevalence of posttransplant hyperparathyroidism: results of an open label randomized trial. Am J Transplant. 2013:13:1576.

7. Trillini M, Cortinovis M, Ruggenenti $P$, et al. Paricacitol for secondary hyperparathyroidism in renal transplantation. J Am Soc Nephrol. 2015;26(5):1205-14.

8. Hadjiyannakos D, Filiopoulos $\mathrm{V}$, Trompouki S, et al. Treatment with oral paricalcitol in daily clinical practice for patients with chronic kidney disease stage 3-4: a preliminary study. Clin Kidney J. 2013:6:164.

9. Cozzolino M, Brancaccio D. Emerging role for the vitamin D receptor activator (VDRA), paricalcitol, in the treatment of secondary hyperparathyroidism. Expert Opin Pharmacother. 2008;9:947.

10. Pihlstrøm HK, Gatti F, Hammarström C. Early introduction of oral paricalcitol in renal transplant recipients. An open-label randomized study. Transpl Int 2017;30:2612-20.

11. Pasch A, Farese S, Grabver S, et al. Nanoparticle-based test measures overall propensity for calcification in serum. J Am Soc Nephrol. 2012;23:1744-52.

12. Smith ER, Ford ML, Tomlinson LA, et al. Serum calcification propensity predicts all-cause mortality in predialysis CKD. J Am Soc Nephrol. 2014:25(2):339-48.

13. .Pasch A, Block GA, Bachtler M et al. Blood calcification propensity, cardiovascular events, and survival in patients receiving hemodialysis in the EVOLVE trial. Clin J Am Soc Nephrol 2017 Feb 7;12(2):315-322. https://doi.org/10.2215/CJN.04720416. Epub 2016 Dec 9.

14. Keyzer CA, de Borst MH, van den Berg E, et al. Calcification Propensity and Survival among Renal Transplant Recipients. J Am Soc Nephrol. 2016:27(1):239-48

15. Dahle DO, Åsberg A, Hartmann A, et al. Serum Calcification Propensity Is a Strong and Independent Determinant of Cardiac and All-Cause Mortality in Kidney Transplant Recipients. Am J Transplant. 2016;16(1):204-12.

16. Smerud KT, Åsberg A, Kile HA. rapid and sustained improvement of calcification propensity score (serum T50 ) after successful kidney transplantation: Reanalysis of a randomized controlled trial of ibandronate. Clin Transpl. 2017 Dec;31(12):e13131. 